\title{
Effect of downhill walking on next-day muscle damage and glucose metabolism in healthy young subjects
}

\author{
Airi Nakayama ${ }^{1} \cdot$ Wataru Aoi $^{1} \cdot$ Maki Takami $^{1} \cdot$ Nariyuki Hirano $^{1} \cdot$ Yumi Ogaya $^{1} \cdot$ Sayori Wada $^{1} \cdot$ Akane Higashi $^{1}$
}

Received: 24 December 2017 / Accepted: 12 April 2018 / Published online: 20 April 2018

(c) The Physiological Society of Japan and Springer Japan KK, part of Springer Nature 2018

\begin{abstract}
This study aimed to investigate the effect of downhill walking on muscle damage and glucose metabolism in healthy subjects. All ten healthy young men and women (age, $24.0 \pm 1.4$ years) performed rest, uphill walking, and downhill walking trials. In the exercise trials, uphill (+5\%) or downhill (-5\%) treadmill walking was performed at $6 \mathrm{~km} / \mathrm{h}$ for $30 \mathrm{~min}$. On the next day, muscle soreness was significantly higher in the downhill trial than in the uphill trial $(P<0.01)$. Respiratory metabolic performance did not differ between trials. However, carbohydrate oxidation was negatively correlated with plasma creatine kinase $(\mathrm{r}=-0.41)$ and muscle soreness $(\mathrm{r}=-0.47)$. Fasting blood glucose was significantly lower in the uphill trial than in the rest trial $(P<0.01)$ but not in the downhill trial. These observations suggest that downhill but not uphill walking causes mild delayed-onset muscle damage, which did not cause marked impairment in glucose metabolism. However, higher muscle damage responders might exhibit lower glucose metabolism.
\end{abstract}

Keywords Walking $\cdot$ Skeletal muscle $\cdot$ Delayed-onset muscle damage $\cdot$ Glucose metabolism $\cdot$ Insulin sensitivity

\section{Introduction}

Exercise induces a drastic change in metabolic events in the body, particularly those such as glucose uptake and lipolysis in the skeletal muscle. Consequently, the energy expenditure during exercise increases above tenfold in the entire body and 100-fold in exercising muscle tissues. A single bout of exercise elevates glucose uptake in skeletal muscle by activating an insulin-independent signal pathway during muscle contraction $[1,2]$. Blood glucose was consumed over $70 \%$ in skeletal muscle. This level was markedly decreased during exercise, which could be attended by even low-intensity exercise such as walking. Previous studies [3,4] showed that brisk walking after meal ingestion decreased the postprandial blood glucose levels by half in healthy subjects and patients with metabolic diseases. Habitual walking in daily life adaptively reduces casual blood glucose by improving insulin sensitivity in the skeletal muscle $[5,6]$; therefore,

Wataru Aoi

waoi@kpu.ac.jp

1 Division of Applied Life Sciences, Graduate School of Life and Environmental Sciences, Kyoto Prefectural University, 1-5 Hangi-cho Shimogamo, Sakyo-ku, Kyoto 606-8522, Japan daily physical activity is advised to prevent and treat noncommunicative diseases.

Acute, unaccustomed, or strenuous exercise causes delayed-onset muscle damage that presents clinically as muscular pain and involves inflammation and ultrastructural changes. Soluble muscle enzymes such as creatine kinase (CK) are released, indicating sarcomere architecture disruption and plasma membrane damage [7-9]. Delayed muscle damage usually occurs after exercise (rather than during or immediately after) and peaks after approximately 1-3 days. Previous studies $[9,10]$ have shown that delayed-onset muscle damage is mainly induced by mechanical stress, particularly eccentric muscle contractions. Concentric exercise shortens contracting muscles; in contrast, eccentric exercise forcibly lengthens contracting muscles. While stepping down a slope, the contracting quadriceps muscle controls the rate of knee flexion against the force of gravity. In this process, the muscle undergoes an eccentric contraction with each step. Thus, compared to uphill running (i.e., concentric exercise), downhill running (i.e., eccentric contraction) causes greater muscle damage and soreness [11, 12].

It is well known that accelerated glucose uptake with improved insulin sensitivity in the skeletal muscles continues for some time after exercise [13, 14]. However, growing evidence has shown that the improvement of insulin sensitivity 
is weakened physiologically with muscle-damaging exercises [15, 16], but not with non-muscle-damaging exercises. Inflammatory cytokines and reactive oxygen species (ROS) that are generated during and after damaging exercise can impair glucose uptake by inhibiting insulin signal transduction in the skeletal muscles $[15,16]$. However, apart from moderate- to high-intensity running or resistance exercise, it is unclear whether and to what degree muscle damage occurs even in low-intensity exercise walking, a major daily physical activity for a wide range of generations that is commonly used in exercise therapy against metabolic diseases. Therefore, we investigated the correlation between walking on different slopes on next-day muscle damage and glucose metabolism in healthy young subjects.

\section{Methods}

\section{Subjects}

Ten healthy young men and women were recruited to participate in this study, which was approved by the Ethics Committee of Kyoto Prefectural University (permission no. 45). All subjects read and signed an informed consent form prior to participation in the study. Each subject was free of signs, symptoms, and a history of any overt chronic disease. None of the subjects were currently taking any medications or dietary supplements, smoking, or exercising regularly. In the first trial, body composition (HBF-361, Omron Healthcare Co., Ltd, Kyoto, Japan) was measured. The subjects' characteristics are shown in Table 1.

\section{Study design}

The subjects participated in three trials consisting of rest, uphill walking, and downhill walking in a repeated-measures design. Each trial was randomly performed over 2 consecutive days and separated by at least 3 weeks in individual subjects to avoid bias. The subjects were also asked to refrain from exertional activity and ingesting supplements, caffeine, or alcohol from the day before each trial.

Table 1 Subjects' characteristics

\begin{tabular}{lrc}
\hline & Male $(n=5)$ & Female $(n=5)$ \\
\hline Age (years) & $25.6 \pm 2.6$ & $22.4 \pm 0.5$ \\
Height $(\mathrm{cm})$ & $172.2 \pm 0.8$ & $158.7 \pm 2.5$ \\
Body weight $(\mathrm{kg})$ & $68.4 \pm 3.5$ & $50.8 \pm 1.7$ \\
Body mass index $\left(\mathrm{kg} / \mathrm{m}^{2}\right)$ & $23.1 \pm 1.0$ & $20.2 \pm 2.1$ \\
Body fat $(\%)$ & $16.1 \pm 1.9$ & $22.7 \pm 1.0$ \\
\hline
\end{tabular}

Values are represented as mean \pm standard error
Their diet on each trial day was recorded and repeated to minimize diet-related bias.

\section{Experiment schedule}

In the walking trials, uphill or downhill walking was performed for $30 \mathrm{~min}$. In the rest trial, the subjects were kept in a resting state during the measurement. On the first experimental day of each trial, the subjects were asked not to eat or drink anything except for water from 22:00 to the measurement the next morning. On the second day, they returned to the laboratory at 9:00 while maintaining their fast, sat on the chair, and rested for $10 \mathrm{~min}$. Subsequently, blood pressure was measured (EW3100, Panasonic Electric Works Co., Ltd., Osaka, Japan) and blood was collected with a finger stick pick. A glucose beverage containing $75 \mathrm{~g}$ of glucose (Trelan ${ }^{\circledR}-\mathrm{G} 75$, Ajinomoto Pharmaceuticals Co. Ltd., Tokyo, Japan) was orally consumed at 9:30, and blood was collected at 10:00 and 10:30. From 10:00, respiratory gas was measured for $30 \mathrm{~min}$ in the supine rest position. Subsequently, subjective muscle pain in the thigh, lower leg, and gluteus maximus, the main muscles exercised during walking, was evaluated by movement (butterfly and squat) described in our previous study [26]. The visual analog scale (VAS) was used to examine the level of muscle pain. Subjects were asked to indicate the intensity of perceived pain for each muscle part on a 100-mm horizontal line. The left side of the scale was designated "no pain", while the right side was designated "max pain." The total soreness value was calculated by adding the soreness values on the three muscle parts. A schematic illustration of the experimental schedule is shown in Fig. 1.

\section{Exercise procedure}

Subjects ingested steamed rice ( $200 \mathrm{~g}$ for men, $150 \mathrm{~g}$ for women) $2 \mathrm{~h}$ before the exercise and then rested on a chair for $20 \mathrm{~min}$. After warm-up with a bicycle ergometer at $30 \mathrm{~W}$ for $5 \mathrm{~min}$, the subjects performed uphill (5\% incline grade) or downhill (5\% decline grade) walking exercise using a treadmill (My Mountain 5050, TOBEONE, Korea) at a speed of $6 \mathrm{~km} / \mathrm{h}$ for $30 \mathrm{~min}$. During exercise, the heart rate was continuously recorded by the heart rate monitor (Polar CS600X ${ }^{\mathrm{TM}}$, Polar Electro, Finland), and exercise intensity was estimated using the Karvonen formula. At the end of the exercise, the rating of perceived exertion (RPE) - the Borg 15-point (6-20) scale - was measured. Immediately after exercise, blood was collected using a finger stick pick and lactate was measured. The degree of subjective fatigue was evaluated using VAS. 
Fig. 1 Schematic illustration of the experimental schedule

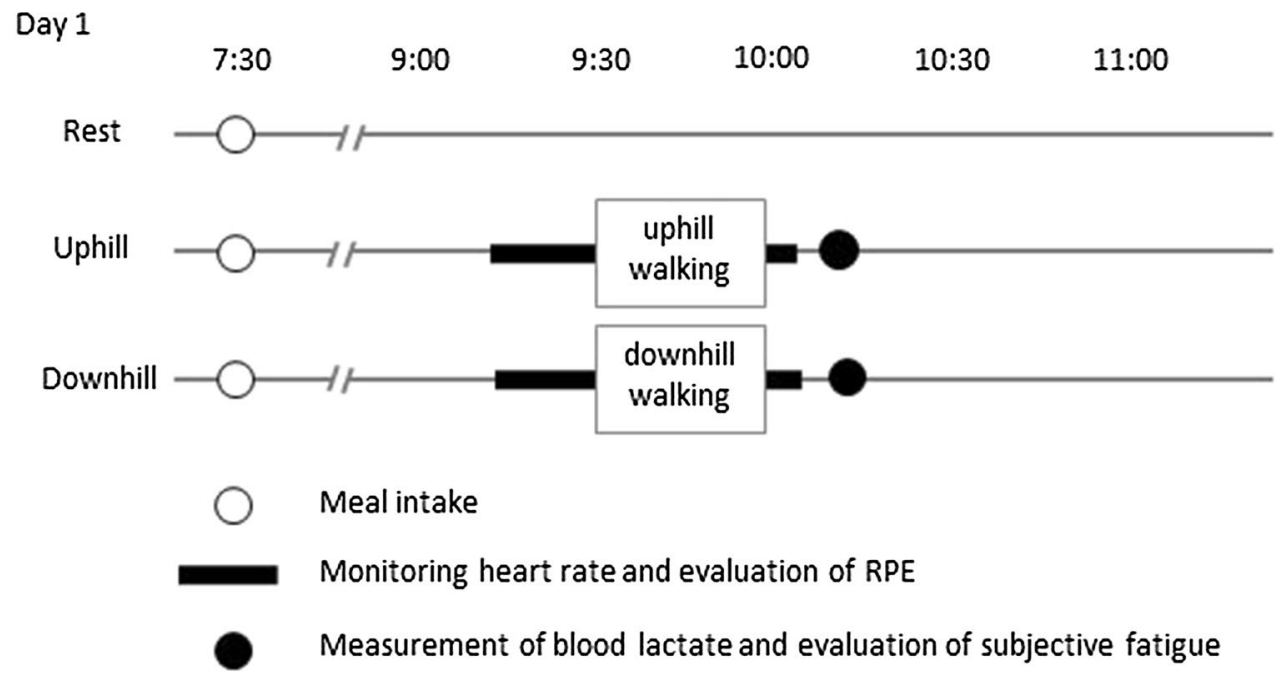

Day 2
$9: 00$ $9: 30$

10:00

$10: 30$

$11: 00$

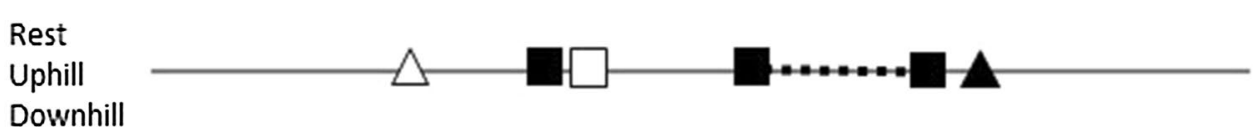

\section{Biochemical analyses}

Blood lactate and glucose were measured using simple measuring instruments (GluTest; Sanwa Kagaku Kenkyusho Co., Ltd., Nagoya, Japan: Lactate Pro; Arkray Inc., Kyoto, Japan). The remaining blood sample obtained during the fasting condition on day 2 of each trial was centrifuged at $3500 \mathrm{rpm}$ to separate the plasma. Plasma CK and insulin levels were each measured using enzyme-linked immunosorbent assay kits (CK; Bioo Scientific Corporation, Austin, TX, USA: insulin; Mercodia AB, Uppsala, Sweden). Homeostasis model assessment-insulin resistance (HOMA-IR), an insulin resistive index, was calculated using the fasting blood glucose and plasma insulin values.

\section{Indirect metabolic performance}

Oxygen consumption $\left(\mathrm{VO}_{2}\right)$ and carbon dioxide production $\left(V \mathrm{CO}_{2}\right)$ were measured by a breath-by-breath respirometer system (AEROMONITOR AE-310S; Minato Medical Science, Ltd, Osaka, Japan). The respiratory quotient (RQ) and substrate utilization were calculated from the $\mathrm{VO}_{2}$ and $V \mathrm{CO}_{2}$ levels as described previously [17].

\section{Statistical analysis}

All data are shown as mean value \pm standard error. Based on normal distribution analysis, non-parametric analysis was used for all parameters. The Friedman test was used to compare between the three trials, while the Steel test was used for multiple comparisons. Differences between two trials were evaluated using a Wilcoxon signed-rank test. The correlation of two variables was analyzed using Spearman's correlation analysis. Significant levels between means were set at $P<0.05$. 
Table 2 Comparison of exercise intensity and fatigue-related parameters between uphill and downhill trials

\begin{tabular}{lccc}
\hline & Uphill & Downhill & $P$ value \\
\hline$\% \mathrm{HR}_{\max }(\%)$ & $48.4 \pm 13.5$ & $26.5 \pm 7.4$ & 0.009 \\
RPE (score) & $12.7 \pm 0.4$ & $12.8 \pm 0.5$ & 0.396 \\
VAS (score) & $5.1 \pm 0.8$ & $3.5 \pm 0.9$ & 0.026 \\
Blood lactate (mmol/l) & $1.4 \pm 0.1$ & $0.8 \pm 0.0$ & 0.006 \\
\hline
\end{tabular}

Values are represented as mean \pm standard error for 10 subjects

The Wilcoxon signed-rank test was used to compare means of the 2 trials

$H R$ heart rate, $R P E$ rating of perceived exertion, $V A S$ visual analog scale

\section{Results}

\section{Fatigue-related parameters on the day of exercise}

Exercise intensity estimated from heart rate at the end of exercise was significantly lower in the downhill trial than in the uphill trial $(P<0.01)$, whereas the RPE score did not differ between trials (Table 2). The degree of subjective fatigue immediately after exercise was significantly lower in the downhill trial than in the uphill trial $(P=0.02)$ (Table 2). The blood lactate level was significantly lower in the downhill trial than in the uphill trial $(P<0.01)$ (Table 2$)$.

\section{Next-day muscle damage parameters and blood pressure}

On the day after exercise, the degrees of muscle soreness in the thigh $(P=0.04)$, lower leg $(P=0.02)$, and gluteus maximus $(P<0.01)$ were significantly higher in the downhill trial than in the uphill trial (Table 3). The total soreness score in the downhill trial was also significantly higher than that in the uphill trial $(P<0.01)$ (Table 3$)$. Although plasma CK level was not significantly changed between trials $(P=0.27)$, the mean value was the highest in the downhill trial (Table 3). Both systolic (rest; $101 \pm 2 \mathrm{mmHg}$, uphill; $98 \pm 3 \mathrm{mmHg}$, downhill; $100 \pm 3 \mathrm{mmHg}$ ) and diastolic (rest, $59 \pm 2 \mathrm{mmHg}$; uphill, $60 \pm 3 \mathrm{mmHg}$; downhill, $61 \pm 2 \mathrm{mmHg}$ ) blood pressures did not change between trials.

\section{Next-day metabolic parameters in the blood}

The next-day fasting blood glucose concentration was significantly lower in the uphill trial than in the rest trial $(P=0.01)$, although this decrease was not found in the downhill trial (Fig. 2a). Area under the curve of blood glucose concentration for $60 \mathrm{~min}$ after glucose administration did not differ between trials (Fig. 2b).

The next-day fasting plasma insulin level did not differ between trials. HOMA-IR was not significantly changed
Table 3 Comparison of plasma $\mathrm{CK}$ and muscle soreness among rest, uphill, and downhill trials
Fig. 2 Blood glucose concentration on the next day of exercise. Fasting blood glucose concentration (a) and area under the curve of the concentration for $60 \mathrm{~min}$ after oral glucose administration (b) were analyzed. Values are represented as mean \pm standard error for 10 subjects. *Statistically significant differences were at the level of $P<0.05$

\begin{tabular}{llccr}
\hline & Rest & Uphill & Downhill & $P$ value \\
\hline Plasma CK (IU/l) & $133.9 \pm 17.3$ & $124.6 \pm 12.8$ & $162.5 \pm 23.4$ & 0.27 \\
Muscle soreness thigh (score) & - & $0.4 \pm 0.2$ & $1.0 \pm 0.4$ & 0.04 \\
Lower leg (score) & - & $0.1 \pm 0.1$ & $1.4 \pm 0.5$ & 0.01 \\
Gluteus (score) & - & $0.3 \pm 0.1$ & $3.3 \pm 0.8$ & $<0.01$ \\
Total (score) & - & $0.9 \pm 0.3$ & $5.8 \pm 1.4$ & $<0.01$ \\
\hline
\end{tabular}

Values are represented as mean \pm standard error for 10 subjects

The Friedman test was used to compare means of the 3 trials and the Wilcoxon signed-rank test was used to compare means of the 2 trials
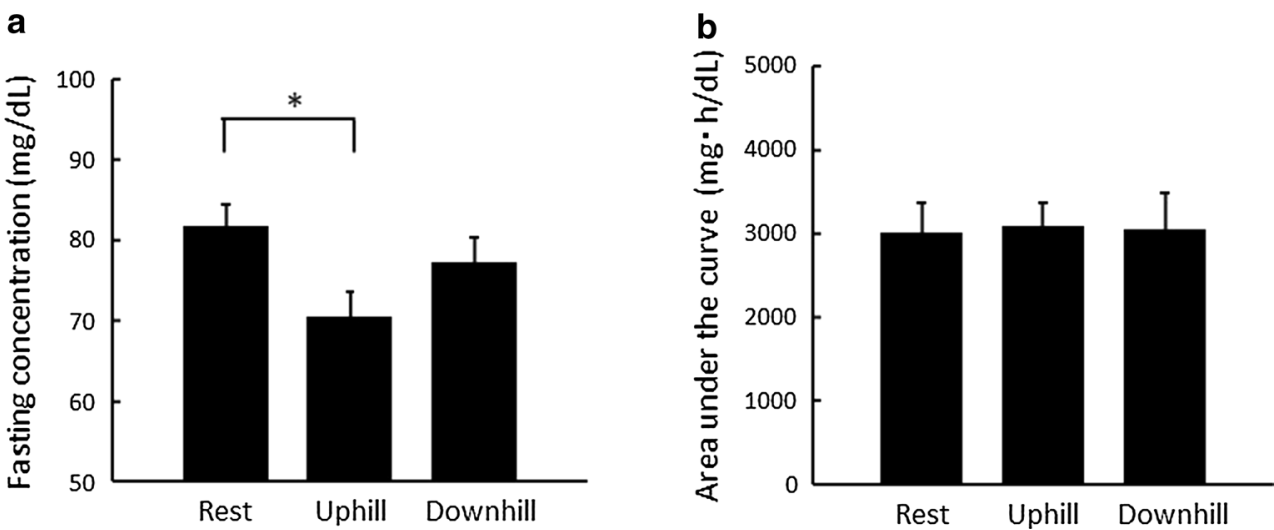
between trials $(P=0.26)$, although the mean value was lower in the exercise trials (Table 4 ).

\section{Indirect metabolic performance}

The mean values of oxygen consumption, RQ, and carbohydrate oxidation did not differ between trials (Table 4). There was a negative correlation between carbohydrate oxidation and plasma $\mathrm{CK}$ ( $\mathrm{r}=-0.41, P=0.014$ ) (Fig. 3a). In addition, there was a tendency toward a negative correlation between carbohydrate oxidation and muscle soreness, with soreness levels above the median value of $1.25(\mathrm{r}=-0.47, P=0.079)$ (Fig. 3b).

\section{Discussion}

The present study examined the effect of different walking exercise slopes on delayed-onset muscle damage. We found that next-day muscle soreness, a muscle damage parameter, was markedly higher in the downhill walking trial than in the uphill walking trial, although heart rate and the fatigue VAS during exercise were lower. These results suggest that downhill walking caused muscle damage regardless of exercise intensity compared to uphill walking. While stepping down a slope, the quadriceps femoris and lower leg triceps muscles actively create an eccentric contraction that provides higher mechanical stimulation to the muscles than the concentric contraction that is caused by stepping up a slope. It was previously shown that high-intensity exercise such as downhill running and resistance exercise markedly induces delayed-onset muscle damage [15, 18]. However, here we show that even low-intensity exercise, i.e., walking, which is a major daily physical activity, could also induce mild muscle damage.

After exercise, the glucose metabolism in the skeletal muscle is improved, continues for several hours after exercise, and often persists until the next day. This is mainly caused by glucose uptake through the insulin-dependent signaling pathway, which involves phosphorylation of the insulin receptor, phosphorylation of the insulin receptor substrate (IRS)-1/2 on its tyrosine residues, and activation of
Table 4 Comparison of metabolic parameters among rest, uphill, and downhill trials

\begin{tabular}{llccc}
\hline & Rest & Uphill & Downhill & $P$ value \\
\hline Oxygen consumption $(\mathrm{ml} / \mathrm{kg} / \mathrm{min})$ & $3.36 \pm 0.08$ & $3.33 \pm 0.06$ & $3.25 \pm 0.06$ & 0.21 \\
Respiratory quotient & $0.88 \pm 0.01$ & $0.87 \pm 0.01$ & $0.88 \pm 0.01$ & 0.84 \\
Carbohydrate oxidation $(\mathrm{mg} / \mathrm{kg} / \mathrm{min})$ & $2.67 \pm 0.16$ & $2.52 \pm 0.19$ & $2.57 \pm 0.14$ & 0.51 \\
Fat oxidation $(\mathrm{mg} / \mathrm{kg} / \mathrm{min})$ & $0.67 \pm 0.05$ & $0.71 \pm 0.07$ & $0.66 \pm 0.05$ & 0.72 \\
Plasma insulin $(\mu \mathrm{U} / \mathrm{ml})$ & $5.6 \pm 1.4$ & $3.5 \pm 0.6$ & $3.4 \pm 0.5$ & 0.24 \\
HOMA-IR & $1.1 \pm 0.3$ & $0.6 \pm 0.1$ & $0.7 \pm 0.1$ & 0.26 \\
\hline
\end{tabular}

Indirect metabolic performance was examined using the mean values during 30 min after glucose administration

The Friedman test was used to compare means of the 3 trials

HOMA-IR homeostasis model assessment-insulin resistance

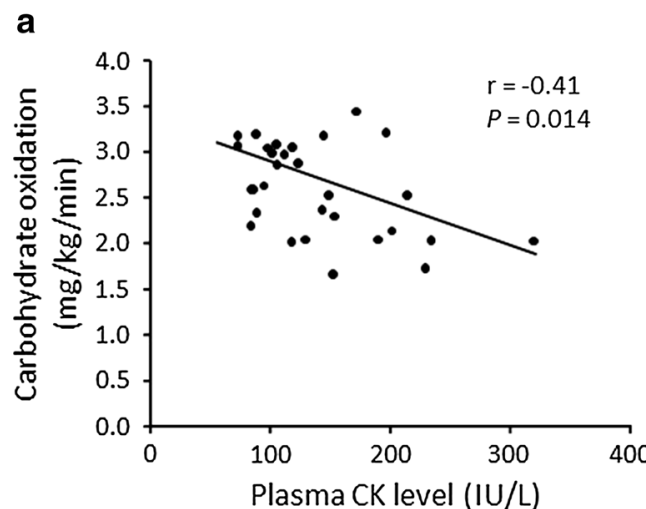

Fig. 3 Correlation between carbohydrate oxidation and muscle damage parameters. a The correlation between carbohydrate oxidation and creatine kinase (CK) was analyzed using Spearman's correlation. The plots from all data obtained from 3 trials are represented. b The

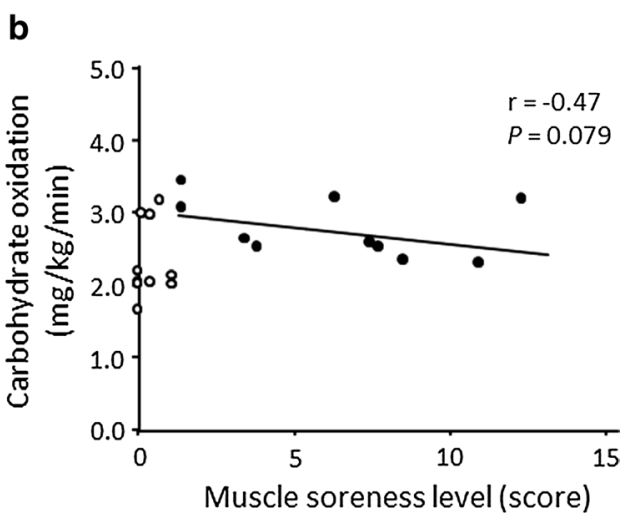

correlation between carbohydrate oxidation and muscle soreness was analyzed for the data showing higher soreness levels. The filled circle plots reflect data above the median soreness value of 1.25 and the open circle plots reflect data below the median value 
phosphatidylinositol 3-kinase (PI3-K) in exercised muscles $[19,20]$. In agreement with this concept, we found a lower level of fasting blood glucose the day after exercise in the uphill walking trial compared to the rest trial, suggesting ameliorated uptake of blood glucose into muscle by simply walking. In contrast, no significant difference was found in the downhill walking trial compared with the rest trial. Moreover, there were negative correlations between muscle damage parameters and carbohydrate oxidation. Specifically, muscle soreness was negatively correlated with carbohydrate oxidation in subjects with higher soreness levels. These observations suggest that there was less improvement in glucose metabolism in the downhill trial and that higher muscle damage responders may exhibit lower glucose metabolism. Therefore, exercise-induced metabolic benefits differ by type and subject characteristics, even with low-intensity exercise.

Cellular inflammatory events may be associated with the ameliorated metabolic benefit in the downhill walking. Previous studies [21, 22] have shown - in animal pathological models and culture experiments - that inflammation and oxidative stress cause insulin resistance in the skeletal muscle. The stimulation of oxidants and tumor necrosis factor-alpha (TNF- $\alpha$ ) easily inactivates insulin signaling in cultured muscle cells [21]. In addition, accumulated oxidative products and higher TNF- $\alpha$ expression were often found in muscle tissues from diabetic mice [23]. Several studies have shown that, after muscle-damaging exercise, phagocytes infiltrating into the muscle tissues generate ROS via nicotinamide adenine dinucleotide phosphate oxidase and myeloperoxidase [24, 25], which causes oxidative damage and accelerates inflammation. We showed that whole-body carbohydrate oxidation after oral glucose administration was decreased the day after acute exercise in healthy young subjects [26]. In addition, we and other researchers have reported - in animal and human experiments - that glucose uptake into damaged muscle after acute running was impaired with suppressed activity of insulin signal molecules, i.e., IRS-1, PI3-K, and Akt $[15,16]$. In the damaged muscle, accumulating oxidative products and increased inflammatory cytokines/ chemokines were found. We showed that IRS-1 modified by 4-hydroxy-2-nonenal (4-HNE), a peroxidation of $n-6$ polyunsaturated fatty acids, was elevated in damaged muscle obtained from mice after acute running, together with reduced tyrosine phosphorylation of IRS-1, inhibition of PI3-K/Akt signaling, and inhibition of the membrane translocation of GLUT4 [16, 27], which suggests that oxidative post-translational modification could inactivate key proteins in the insulin signal pathway. In addition, levels of interleukin (IL)-1 $\beta$, IL-6, monocyte chemoattractant protein-1, and IL-8 are increased in muscle tissues 24-48 $\mathrm{h}$ after muscle-damaging exercises in humans and rodents $[24,28,29]$. These oxidative and inflammatory factors may be partially associated with the initiation and progression of impaired muscle damage-induced insulin signaling.

It is widely known that daily exercise contributes to the prevention and improvement of non-communicative diseases such as metabolic syndrome, diabetes, hypertension, and cancer. However, muscle-damaging exercise could cause several negative events related to athletic performance and health promotion. In addition to impaired glucose metabolism, reduced force generation [30] and elevated arterial stiffness [31] occur in subjects with delayed-onset muscle damage. The present study shows that muscle damage could occur even after lowintensity exercise, specifically downhill walking, which suggests that walking on a flat plane or uphill can be recommended more as an exercise for health promotion, depending on individual physical performance level and exercise purpose. However, there were several limitations in this study. First, the intensity and volume of the walking exercise were low to obtain enough metabolic changes and muscle damage in young healthy subjects. For example, it is known that insulin sensitivity in skeletal muscle is improved with intensity and duration of exercise $[32,33]$. In contrast, muscle damage-induced metabolic impairment may easily occur in middle-aged to elderly people and metabolic patients. Second, we confirmed that all female subjects had normal estrous cycle, and their trials were performed in the same estrous cycle within the individual, although the cycle was not consolidated between participants. Circulating estradiol and progesterone levels might affect energy metabolism in both basal and exercise conditions. The response of muscle damage to exercise could also be different between males and females [34]. Third, metabolic and damage parameters post exercise were examined only $24 \mathrm{~h}$ after exercise. Because the peaks of blood damage parameters and soreness are often different depending on the conditions of exercise and subjects, time-course examination would give more information. Finally, dietary status before and after exercise also affects metabolic and inflammatory responses to exercise. Before exercise, study subjects consumed steamed rice, a Japanese dietary staple, because exercise after prolonged fasting is not recommended. However, lunch and dinner after exercise were not strictly controlled, possibly affecting muscle damage and metabolic parameters on the following day. Thus, further research is required to examine whether these findings can be generalized to the larger public under strict experimental conditions. In addition, it is important to survey, in addition to healthy subjects, various populations like elderly people and patients with metabolic disorders, in order to define appropriate exercise methods for promoting health and managing muscle damage. 


\section{Conclusion}

We found that muscle soreness was markedly higher in the downhill trial than in the uphill trial. Fasting blood glucose was significantly lower in the uphill trial than in the sedentary trial, but this phenomenon was not seen in the downhill trial. There were negative correlations between carbohydrate oxidation and muscle damage parameters. Downhill walking causes mild delayed-onset muscle damage, which did not cause significant impairment in glucose metabolism. However, higher muscle damage responders may exhibit lower glucose metabolism after exercise.

Acknowledgements This work was partly supported by the Japan Society for the Promotion of Science (JSPS) KAKENHI: Grant-in-Aid for Scientific Research (B) Grant numbers 25282199 and 17H02176.

\section{Compliance with ethical standards}

Conflicts of interest The authors declare no conflict of interest.

Ethical approval All procedures performed in studies involving human participants were in accordance with the ethical standards of the institutional and/or national research committee and with the 1964 Helsinki Declaration and its later amendments or comparable ethical standards. All the participants signed an informed consent form agreeing to submit to the procedures involved in the study.

\section{References}

1. Fisher JS, Gao J, Han DH, Holloszy JO, Nolte LA (2002) Activation of AMP kinase enhances sensitivity of muscle glucose transport to insulin. Am J Physiol Endocrinol Metab 282(1):E18-E23

2. Wright DC, Hucker KA, Holloszy JO, Han DH (2004) $\mathrm{Ca}^{2+}$ and AMPK both mediate stimulation of glucose transport by muscle contractions. Diabetes 53(2):330-335

3. Manohar C, Levine JA, Nandy DK, Saad A, Dalla Man C, McCrady-Spitzer SK, Basu R, Cobelli C, Carter RE, Basu A, Kudva YC (2002) The effect of walking on postprandial glycemic excursion in patients with type 1 diabetes and healthy people. Diabetes Care 35(12):2493-2499

4. Lunde MS, Hjellset VT, Høstmark AT (2012) Slow post meal walking reduces the blood glucose response: an exploratory study in female Pakistani immigrants. J Immigr Minor Health 14(5):816-822

5. Miyatake N, Nishikawa H, Morishita A, Kunitomi M, Wada J, Suzuki H, Takahashi K, Makino H, Kira S, Fujii M (2002) Daily walking reduces visceral adipose tissue areas and improves insulin resistance in Japanese obese subjects. Diabetes Res Clin Pract 58(2):101-107

6. Peterson MJ, Morey MC, Giuliani C, Pieper CF, Evenson KR, Mercer V, Visser M, Brach JS, Kritchevsky SB, Goodpaster BH, Rubin S, Satterfield S, Simonsick EM, Health ABC Study (2010) Walking in old age and development of metabolic syndrome: the health, aging, and body composition study. Metab Syndr Relat Disord 8(4):317-322

7. Friden J, Sjostrom M, Ekblom B (1981) A morphological study of delayed muscle soreness. Experientia 37(5):506-507
8. Friden J, Sjostrom M, Ekblom B (1983) Myofibrillar damage following intense eccentric exercise in man. Int J Sports Med 4(3):170-176

9. Newham DJ, Jones DA, Edwards RH (1983) Large delayed plasma creatine kinase changes after steppimg exercise. Muscle Nerve 6(5):380-385

10. Kyröläinen H, Takala TE, Komi PV (1998) Muscle damage induced by stretch-shortening cycle exercise. Med Sci Sports Exerc 30(3):415-420

11. Malm C, Sjödin TL, Sjöberg B, Lenkei R, Renström P, Lundberg IE, Ekblom B (2004) Leukocytes, cytokines, growth factors and hormones in human skeletal muscle and blood after uphill or downhill running. J Physiol 556(3):983-1000

12. Imaoka Y, Kawai M, Mori F, Miyata H (2015) Effect of eccentric contraction on satellite cell activation in human vastus lateralis muscle. J Physiol Sci 65(5):461-469

13. Richter EA, Garetto LP, Goodman MN, Ruderman NB (1982) Muscle glucose metabolism following exercise in the rat: increased sensitivity to insulin. J Clin Invest 69(4):785-793

14. Treebak JT, Frøsig C, Pehmøller C, Chen S, Maarbjerg SJ, Brandt N, MacKintosh C, Zierath JR, Hardie DG, Kiens B, Richter EA, Pilegaard H, Wojtaszewski JF (2009) Potential role of TBC1D4 in enhanced post-exercise insulin action in human skeletal muscle. Diabetologia 52(5):891-900

15. Del Aguila LF, Krishnan RK, Ulbrecht JS, Farrell PA, Correll PH, Lang CH, Zierath JR, Kirwan JP (2000) Muscle damage impairs insulin stimulation of IRS-1, PI 3-kinase, and Aktkinase in human skeletal muscle. Am J Physiol Endocrimol Metab 279(1):E206-E212

16. Aoi W, Naito Y, Tokuda H, Tanimura Y, Oya-Ito T, Yoshikawa $\mathrm{T}$ (2012) Exercise-induced muscle damage impairs insulin signaling pathway associated with IRS-1 oxidative modification. Physiol Res 61(1):81-88

17. Frayn KN (1983) Calculation of substrate oxidation rates in vivo from gaseous exchange. J Appl Physiol 55(2):628-634

18. Nogueira FR, Libardi CA, Nosaka K, Vechin FC, Cavaglieri CR, Chacon-Mikahil MP (2014) Comparison in responses to maximal eccentric exercise between elbow flexors and knee extensors of older adults. J Sci Med Sport 17(1):91-95

19. Goodyear LJ, Giorgino F, Balon TW, Condorelli G, Smith RJ (1995) Effects of contractile activity on tyrosine phosphoproteins and phosphatidylinositol 3-kinase activity in rat skeletal muscle. Am J Physiol 268(5 Pt 1):E987-E995

20. Wojtaszewski JF, Hansen BF, Gade Kiens B, Markuns JF, Goodyear LJ, Richter EA (2000) Insulin signaling and insulin sensitivity after exercise in human skeletal muscle. Diabetes 49(3):325-331

21. Del Aguila LF, Claffey KP, Kirwan JP (1999) TNF-alpha impairs insulin signaling and insulin stimulation of glucose uptake in C2C12 muscle cells. Am J Physiol Endocrimol Metab 276(5 Pt 1):E849-E855

22. Singh I, Carey AL, Watson N, Febbraio MA, Hawley JA (2008) Oxidative stress-induced insulin resistance in skeletal muscle cells is ameliorated by gamma-tocopherol treatment. Eur J Nutr 47(7):387-392

23. Bonnard C, Durand A, Peyrol S, Chanseaume E, Chauvin MA, Morio B, Vidal H, Rieusset J (2008) Mitochondrial dysfunction results from oxidative stress in the skeletal muscle of dietinduced insulin-resistant mice. Clin Invest 118(2):789-800

24. Aoi W, Naito Y, Takanami Y, Kawai Y, Sakuma K, Ichikawa H, Yoshida N, Yoshikawa T (2004) Oxidative stress and delayedonset muscle damage after exercise. Free Radic Biol Med 37(4):480-487

25. Wang HJ, Pan YX, Wang WZ, Zucker IH, Wang W (2009) NADPH oxidase-derived reactive oxygen species in skeletal muscle modulates the exercise pressor reflex. J Appl Physiol 107(2):450-459 
26. Iwasa M, Aoi W, Mune K, Yamauchi H, Furuta K, Sasaki S, Takeda K, Harada K, Wada S, NakamuraY Sato K, Higashi A (2013) Fermented milk improves glucose metabolism in exerciseinduced muscle damage in young healthy men. Nutr J 12:83

27. Aoi W, Naito Y, Sakuma K, Kuchide M, Tokuda H, Maoka T, Toyokuni S, Oka S, Yasuhara M, Yoshikawa T (2003) Astaxanthin limits exercise-induced skeletal and cardiac muscle damage in mice. Antioxid Redox Signal 5(1):139-144

28. Hamada K, Vannier E, Sacheck JM, Witsell AL, Roubenoff R (2005) Senescence of human skeletal muscle impairs the local inflammatory cytokine response to acute eccentric exercise. FASEB J 19(2):264-266

29. Davis JM, Murphy EA, Carmichael MD, Zielinski MR, Groschwitz CM, Brown AS, Gangemi JD, Ghaffar A, Mayer EP (2007) Curcumin effects on inflammation and performance recovery following eccentric exercise-induced muscle damage. Am J Physiol Regul Integr Comp Physiol 292(6):R2168-R2273
30. Clarkson PM, Nosaka K, Braun B (1992) Muscle function after exercise-induced muscle damage and rapid adaptation. Med Sci Sports Exerc 24(5):512-520

31. Barnes JN, Trombold JR, Dhindsa M, Lin HF, Tanaka H (2010) Arterial stiffening following eccentric exercise-induced muscle damage. J Appl Physiol (1985) 109(4):1102-1108

32. Langfort J, Budohoski L, Newsholme EA (1988) Effect of various types of acute exercise and exercise training on the insulin sensitivity of rat soleus muscle measured in vitro. Pflugers Arch 412(1-2):101-105

33. Black LE, Swan PD, Alvar BA (2010) Effects of intensity and volume on insulin sensitivity during acute bouts of resistance training. J Strength Cond Res 24(4):1109-1116

34. Amorim MZ, Machado M, Hackney AC, de Oliveira W, Luz CP, Pereira R (2014) Sex differences in serum CK activity but not in glomerular filtration rate after resistance exercise: is there a sex dependent renal adaptative response? J Physiol Sci 64(1):31-36 